\title{
The physics of rotational tunneling: hole-burning spectroscopy of methyl groups
}

\author{
Mark M. Somoza and Josef Friedrich \\ Physik-Department E14 and Lehrstuhl für Physik Weihenstephan \\ Technische Universität München, Freising, 85350 Germany \\ E-mail: J.Friedrich@lrz.tu-muenchen.de
}

Received February 13, 2006, revised May 29, 2006.

\begin{abstract}
Methyl groups are most outstanding quantum systems due to their inherent symmetry properties which cannot be destroyed by any kind of lattice disorder. We show how optical hole-burning techniques can be employed to measure rotational tunneling relaxation processes. Since the tunneling parameters are extremely sensitive to changes in the host lattice, there is a rich variety of relaxation phenomena that can be observed. Hole-burning techniques have the capability of measuring not only extremely slow processes with high precision but also rather fast processes. We exploit this possibility to show that the relaxation times at $2 \mathrm{~K}$ change by 14 orders of magnitude if the permutation symmetry of the methyl group is destroyed by asymmetric deuterium substitution.
\end{abstract}

PACS: 33.15.Hp, 42.50.Ct, 42.62.Fi, 78.55.Kz

Keywords: rotational tunneling relaxation, nuclear spin polarization, symmetry species conversion, substitution of hydrogen by deuterium in the methyl group.

\section{Introduction}

Let us consider a methyl group attached to a chromophore. A rotation by 120 degrees leaves the methyl group invariant. However, such a rotation is inevitably associated with an even permutation of the hydrogens, i.e. the $C_{3}$-group is isomorphic to the symmetry group of even permutations. Clearly, irrespective of fluctuations of the host lattice or of structural disorder, this symmetry cannot be affected $[1,2]$. It imposes severe quantum effects on the dynamics of the tunneling states: since the nuclear spin is the only property by which the hydrogens can be distinguished, any interaction which induces rotational tunneling relaxation processes must include the nuclear spin. Since nuclear spin interactions are extremely weak, rotational tunneling relaxation times extend to days and weeks at sufficiently low temperatures.

There are various techniques to measure relaxation processes of rotational tunneling states [3-9]. However, it seems that the most accurate technique is optical hole-burning. Hole-burning can be used if two conditions are met: first, the methyl group has to be attached to a chromophoric system so that it can be addressed with light from a high resolution laser, and second, the rotational tunneling splitting in the electronic ground state must be different from the respective one in the electronic excited state so that the optical transitions connecting the various tunneling states can be distinguished by their frequencies. Since the tunneling splitting is tiny compared to optical frequencies, high resolution techniques are required.

The first experiment in which optical transitions associated with rotational tunneling states of a methyl group could be measured, was performed in 1990 [10]. The first relaxation experiments were published in $1992[11,12]$.

In this paper we present an overview of our holeburning experiments on the rotational tunneling states of methyl groups and their dynamic behavior.

\section{Basic physics of a methyl group interacting with a chromophoric system}

Let us discuss the basic physics associated with rotational tunneling by considering the protonated 


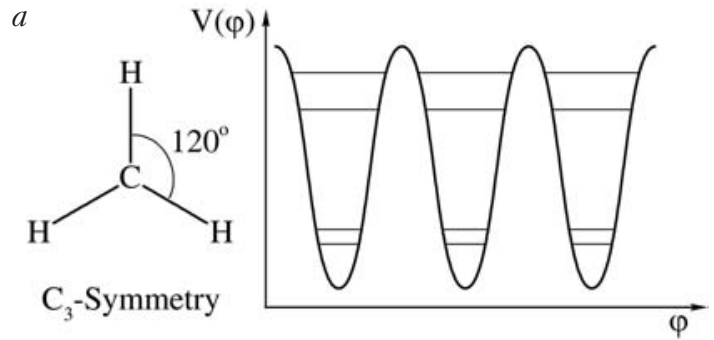

$b$

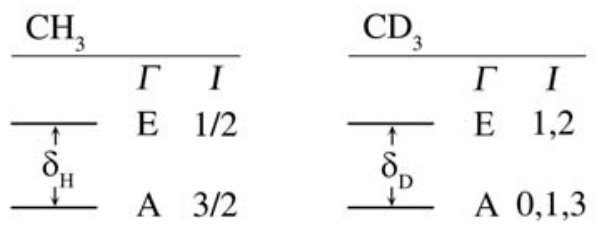

Fig. 1. The methyl group viewed along the 3 -fold rotation axis together with the rotational potential $V=V_{3} \cos (3 \varphi)$. The ground state and the 1st librational excitation is indicated $(a)$. Tunneling splitting, $\delta_{\mathrm{H}}, \delta_{\mathrm{D}}$, of the $\mathrm{CH}_{3}$ and the $\mathrm{CD}_{3}$ group, respectively, together with the symmetry classification and associated total nuclear spin $(b)$.

methyl group first (Fig. 1). As mentioned above, a rotation by 120 degrees is inevitably associated with an even permutation of the protons. Pauli's principle tells us that an even permutation of spin $1 / 2$-particles leaves the respective wavefunction invariant, hence must belong to the totally symmetric representation. To achieve this, the orbital part of the rotor wavefunction and the respective nuclear spin part have to belong to the same representation so that their direct product is totally symmetric. This requirement leads to a strict correlation between rotor wavefunction and nuclear spin: the $A$-symmetric rotor wavefunction correlates with a nuclear spin of $3 / 2$ whereas the $E$-symmetric part correlates with a nuclear spin of $1 / 2$. Since a relaxation within the rotor states necessarily changes the total nuclear spin, the respective processes are called spin conversion. As mentioned in the introduction, any interaction which induces relaxation between the tunneling states must break the permutation symmetry. For the $\mathrm{CH}_{3}$-group, the dominant interaction is the nuclear spin-spin interaction. However, as we will see, for methyl groups attached to organic chromophores, the hyperfine interaction in the excited triplet state is also very important as it has symmetry breaking properties.

The methyl rotor experiences an interaction potential, partly through the electrons of the attached chromophore, and partly through interactions with the host matrix. The potential must at least have 3-fold symmetry, but higher Fourier components are possible as well. However, in most cases, a 3-fold symmetry is a good description. The situation is shown schematically in Fig. 1. The degeneracy of the 3 rotational states is partly lifted because the respective wavefunctions overlap. The $E$-symmetric state is doubly degenerate. The splitting between the $A$ - and $E$-symmetric states, $\delta$, is called the rotational tunneling splitting. This is the most important system parameter in the rotational tunneling dynamics of methyl groups. In the case of a 3 -fold hindering potential, $\delta$ is solely determined by the amplitude $V_{3}$ of the potential. For a quantitative evaluation, the Mathieu equation (Eq. (1)) must be solved. The magnitude of the hindering potential is rather small. The largest splitting occurs for the free rotor. In this case it is determined by the rotational constant, $B=\hbar^{2} / 2 I$, where $I$ is the moment of inertia with respect to the bond linking the methyl group to the chromophore. For $\mathrm{CH}_{3}$ the rotational constant is $5.22 \mathrm{~cm}^{-1}$. If the methyl group experiences a hindering potential, the splitting drops quickly with increasing barrier height.

The situation for the $\mathrm{CD}_{3}$-group is more complicated. On the one hand, this is due to the fact that deuterons are spin 1-particles. As a consequence there is a much larger space of nuclear states. The main consequence is that the correlation between rotor and spin states breaks down because the eigenstates of total spin 1 can be $E$ - as well as $A$-symmetric. On the other hand, and more important as far as relaxation is concerned, deuterons have a nuclear quadrupole moment. The nuclear quadrupole interaction also has symmetry breaking properties and gives rise to peculiar and unexpected isotope effects.

So far we have not discussed how optical spectroscopy can be used to investigate the dynamics of the methyl rotor. To use optical techniques, the methyl group must interact sufficiently with the $\pi$-electrons of the attached chromophore. However, this is not enough. The tunneling splitting in the ground state, $\delta$, has to be sufficiently different from the respective one in the excited state, $\delta^{*}$. Otherwise, nothing can be seen at all. Figure 2 illustrates the situation: suppose the rotor is in its $A$-state when the molecule is in the electronic ground state. Excitation to $S_{1}$, of course, does not change the rotor state, because the nuclear spin is conserved. Since organic molecules may have a large triplet yield, much of the population of the excited state may pass through $T_{1}$. In $T_{1}$ there is a strong (compared to the nuclear spin-spin interaction) hyperfine interaction which may also affect the methyl protons if the delocalization of the electron wavefunction is sufficiently large. If so, conversion from the $A$ - to the $E$-rotor state may be strongly 


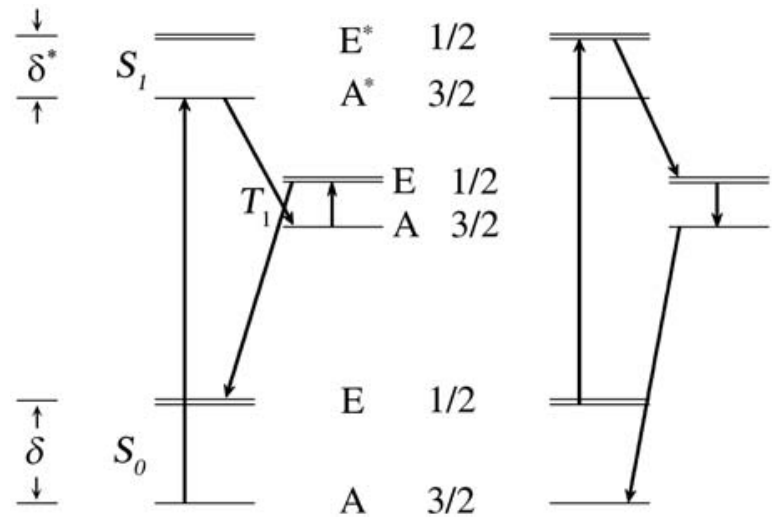

Fig. 2. Optical pumping scheme of the rotational tunneling states of a methyl rotor attached to a chromophore. Relaxation between the tunneling states occurs in $T_{1}$ due to the large hyperfine interaction. An $A-A^{*}$ excitation creates a red shifted, an $E-E^{*}$ excitation creates a blue shifted antihole, since, as a rule, $\delta^{*}<\delta$.

enhanced in $T_{1}$. When the molecule returns to the ground state, it will end up in the $E$-state. Hence, we have optically pumped the tunneling levels from $A$ to $E$. Of course, the reverse occurs as well. In case $\left|\delta-\delta^{*}\right|$ is larger than the optical line width, the two transitions can, in principle, be resolved. The problem is inhomogeneous line broadening. It may be orders of magnitude larger than the difference of the tunneling splitting. This is where hole-burning comes in: at low temperature the tunneling states can live for hours, hence, population can be stored in them. Then, optical pumping removes population, say from the frequency associated with the $A$-transition and piles it up at the frequency associated with the $E$-transition. Of course, the reverse occurs as well, because inhomogeneous broadening leads to an accidental degeneracy of $A-A^{*}$ and $E-E^{*}$ transitions. As a consequence, a hole appears at the laser frequency and, symmetrically shifted by $\left|\delta-\delta^{*}\right|$, two antiholes appear as well. The requirement that the tunneling states can be seen in the spectrum is that the difference $\left|\delta-\delta^{*}\right|$ is larger than the homogeneous linewidth, which is the resolution limit of the hole-burning technique. At $4 \mathrm{~K}$ an order of magnitude number is $1 \mathrm{GHz}$.

\section{Rotational tunneling relaxation processes}

For rotational tunneling processes to occur two requirements have to be met: first, there must be a symmetry breaking interaction. As we have stressed above, such an interaction necessarily involves the nuclear spin of the methyl hydrogens. Second, the system must conserve energy. Energy is conserved by the exchange of phonons with the lattice in which the tunneling system is embedded. We can distinguish three types of processes by their phonon exchange mechanisms which manifest themselves by specific dependencies on temperature: there is the so-called direct process characterized by resonant absorption or emission of a phonon by the tunneling system. The rate of this process depends on the number of thermal phonons with the proper energy. This number scales linearly with $T$, if $T>>\delta$. If $T \ll \delta$, the rate is determined through spontaneous emission and, hence, becomes independent of $T$. Then, there is the so-called Raman process, which is characterized by the exchange of two phonons in a way such that their energy difference matches the energy of the tunneling states. In this case, all phonons in the entire spectrum participate in the relaxation since resonance is not required. This is reflected in a steep temperature dependence of the respective rate, namely $\sim T^{7}$. Finally, there is the so-called Orbach process which is also a two phonon scattering process off a real state within the phonon bath. Accordingly, the temperature dependence is that of an activated process. As a rule, the activation energy is given by the 1st librational excitation of the methyl rotor. The theory of rotational tunneling relaxation was developed by Häusler [13], Würger [14], and Diezemann et al. [15].

\section{A few details of the experiment}

\section{Systems investigated}

The requirement of a sufficiently large value for $\left|\delta-\delta^{*}\right|$ limits the probe molecules to small chromophoric systems, e.g., benzene derivatives. The reason is that the coupling of the methyl group to the $\pi$-electrons roughly scales with the MO-coefficient at the substitution position. Since this coefficient scales with the number of $\pi$-electrons, approximately as $1 / \mathrm{N}^{1 / 2}$, smaller systems are expected to have a larger coupling. As a matter of fact, only two probe molecules have been investigated so far, namely dimethyl-s-tetrazine (DMST- $h_{6}$, DMST- $d_{6}$, DMST- $h_{4} d_{2}$ ) and $p$-chlorotoluene (РCT). However, for the host materials there is little restriction. Quite a series of $n$-alkane matrices, so-called Shpol'skii systems, protonated as well as deuterated, were used. Cyclohexane as a host material was used for PCT. Guest molecules and host systems are shown in Fig. 3.

DMST is a special case because it has two methyl groups. Although these groups are equivalent in the isolated molecule, this is not necessarily the case in a host lattice. Indeed, in some host materials, the two groups give rise to a quite peculiar hole-burning pattern as well as an associated peculiar relaxation pattern. Finally, a forthcoming idea is to lift the 


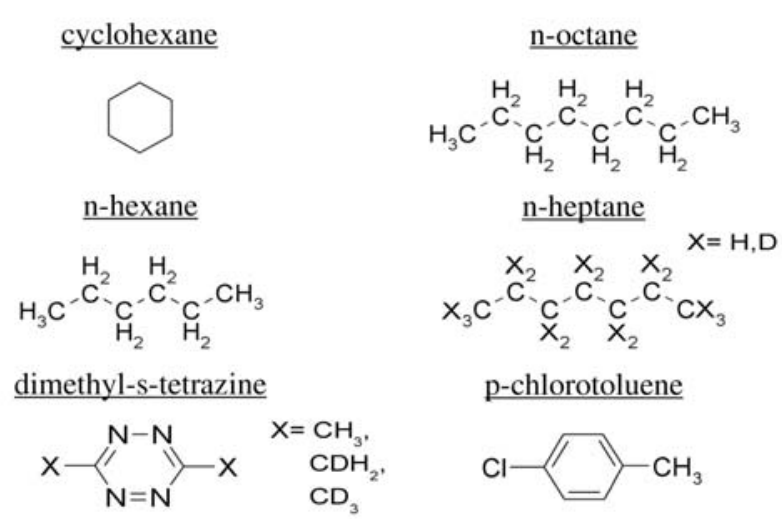

Fig. 3. Probe molecules and host materials used in our hole-burning experiments on rotational tunneling dynamics.

symmetry restrictions by chemically substituting one of the protons with a deuteron.

\section{Hole-burning spectroscopy}

We do not intend to go into too many details regarding hole-burning experiment itself as there are numerous reviews on this method, see for instance, Refs. 16-18. Briefly, the sample is irradiated with a narrow bandwidth laser. A subsequent laser scan of the appropriate frequency range detects a hole at the initial laser frequency if a frequency converting processes took place. The scan width in most cases was $30 \mathrm{GHz}$. For the $p$-chlorotoluene experiments in the UV, where we employed frequency doubling via an external ring resonator, the scan covered $60 \mathrm{GHz}$ or more. The width of the laser radiation was of the order of $1 \mathrm{MHz}$. Laser power levels ranged from about $100 \mu \mathrm{W}$ to $10 \mathrm{~mW} / \mathrm{cm}^{2}$, burning times from about 1 to 10 minutes. The concentrations were chosen in a way that the absorbances were in a range from about $0.1-0.8$.

We mentioned above that the hole-burning reaction may just be the optical pumping from the $A$-symmetric to the $E$-symmetric tunneling state and vice versa. However, as was already pointed out in the very first experiment by Borzcyskowski et al. [10], a photochemical transformation can also serve to detect rotational tunneling splitting. Since the associated lifetimes become extremely long with falling temperature, the rotational tunneling states fall out of equilibrium at some temperature, say $T_{0}$. As a consequence, relaxation processes do occur at $T<T_{0}$ in order to establish equilibrium. Since a conversion process from $A$ to $E$ is correlated with a frequency shift $\left|\delta-\delta^{*}\right|$, the hole will be filled in by relaxing molecules originating at this frequency shift from the hole. The relaxation of these molecules will, in turn, cause a population depletion at frequency distances of $\left|\delta-\delta^{*}\right|$. Hence, as the central photochemical hole is filled, symmetrically shifted side holes may grow in.

The rotational tunneling dynamics is easily measured using hole-burning techniques. One just observes how the central hole recovers, or how the antiholes decay, or how the side holes grow in.

The hole-burning experiment only measures the difference $\left|\delta-\delta^{*}\right|$. The actual splitting $\delta$ can be obtained by solving the Mathieu equation, i.e., the Schrödinger equation for the hindered rotor (see, e.g., Refs. 1, 19). For a 3-fold potential it contains just one parameter, namely the height of the potential barrier, $2 V_{3}$ :

$$
\left(-B \frac{\partial^{2}}{\partial \varphi^{2}}+V_{3} \cos 3 \varphi\right) \psi(\varphi)=E \psi(\varphi) .
$$

For PCT we have shown that although the experiment is only sensitive to the difference between the tunneling splittings, it is possible to extract the individual parameters separately from the experiment (see below).

\section{Measuring the rotational tunneling dynamics}

In the following we present some of our results on the dynamics of methyl groups.

\section{DMST in n-hexane}

Figure 4 shows the absorption spectrum of DMST- $h_{6}$ in $n$-hexane. Judging from the low background in the spectrum, DMST fits perfectly into the lattice. This is also confirmed by Stark spectroscopy: the probe retains its inversion symmetry. The $\mathrm{CH}_{3}$ groups cannot be distinguished. All hole-burning experiments are done in the red-most band, the electronic origin.

Figure 5 shows the recovery rate of the central hole overlaid with data points from the decay rate of the antiholes as a function of temperature. The two data sets closely follow each other, confirming that antiholes and the central hole are governed by the same relaxation process.

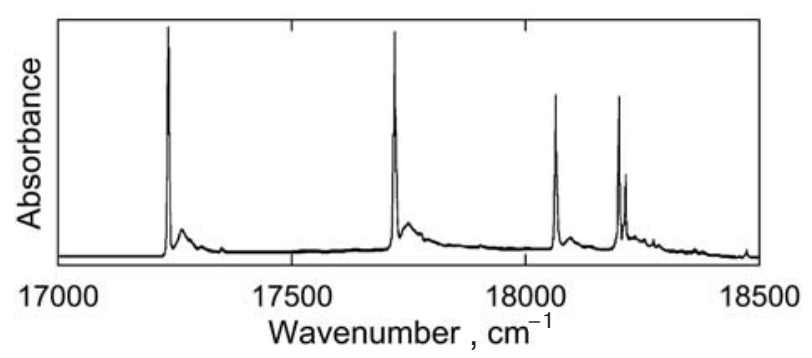

Fig. 4. Absorption spectrum of DMST- $h_{6}$ in $n$-hexane at $4.2 \mathrm{~K}$. 


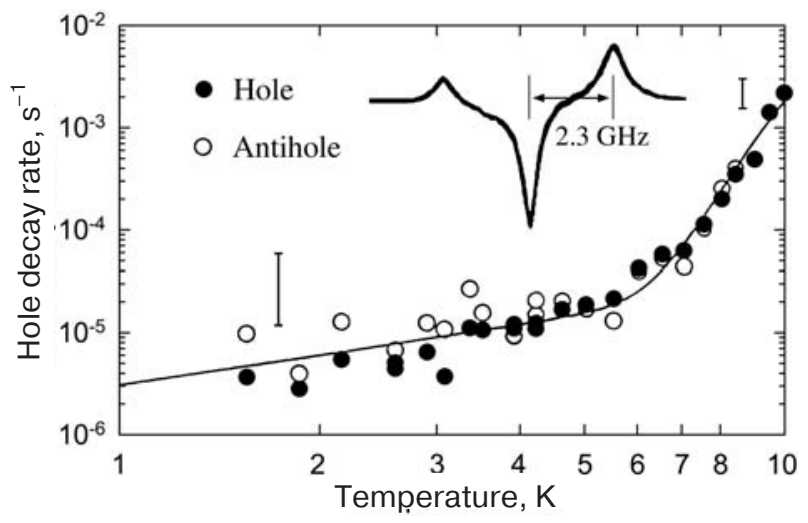

Fig. 5. Tunneling relaxation rate of DMST- $h_{6}$ in $n$-hexane as a function of temperature. Measured was the recovery rate of the central hole (filled symbols) as well as the decay rate of the antiholes (open symbols). The inset shows hole and antiholes at $3.5 \mathrm{~K}$.

Below about $6 \mathrm{~K}$ the rate depends weakly and linearly on temperature, supporting a direct process. Around $6 \mathrm{~K}$ there is a transition to a steep temperature dependence which we associate with an Orbach process. To put this assignment on solid ground we solved the Mathieu equation for the electronic ground state, as well as for the excited state, in order to determine $\delta$ and $\delta^{*}$. Note that the complete set of parameters $V_{3}$, $V_{3}^{*}, \delta_{\mathrm{H}}, \delta_{\mathrm{H}}^{*}, \delta_{\mathrm{D}}, \delta_{\mathrm{D}}^{*}$ are completely determined by the experimentally accessible quantities $\left|\delta_{\mathrm{H}}-\delta_{\mathrm{H}}^{*}\right|$ and $\left|\delta_{\mathrm{D}}-\delta_{\mathrm{D}}^{*}\right|$ where the $\mathrm{H}$ and $\mathrm{D}$ subscripts refer to the $\mathrm{CH}_{3}$ - and $\mathrm{CD}_{3}$-group of DMST, respectively. We also assumed that $V_{3}$ and $V_{3}^{*}$ do not depend on deuteration of the guest molecule in the $n$-hexane host. Data for DMST- $d_{3}$ in $n$-hexane are not shown. With these assumptions all the matrix elements, sums over states, etc., can be calculated. The corresponding parameter-free fit curve (except for a scaling constant) is very close to the experimental one in Fig. 5. The activation energy for the Orbach process is $57 \mathrm{~cm}^{-1}$. The prediction from theory is $60 \mathrm{~cm}^{-1}$. Also the crossover temperature $T_{c}$ in the experiment $(5 \mathrm{~K})$ matches the theoretical prediction $\left(T_{c}=4 \delta_{\mathrm{H}} / \mathrm{k}_{B} \approx\right.$ $\approx 4 \mathrm{~K})$ very well. The energy splittings $\delta_{\mathrm{H}}, \delta_{\mathrm{H}}^{*}, \delta_{\mathrm{D}}, \delta_{\mathrm{D}}^{*}$ are 20.8, 18.5, 1.40 and $1.16 \mathrm{GHz}$, respectively [20].

\section{DMST in n-heptane}

We investigated DMST $-h_{6}$ and DMST- $d_{6}$ in $n$-heptane- $h_{16}$, and DMST- $h_{6}$ also in $n$-heptane- $d_{16}$ [21].

We first discuss the behavior of DMST- $h_{6}$. Figure 6 shows the absorption spectrum in $n$-heptane- $h_{16}$. Again, all hole-burning experiments were carried out in the red-most band. Figure 7 shows a Stark-effect experiment on the central hole. The hole splits under

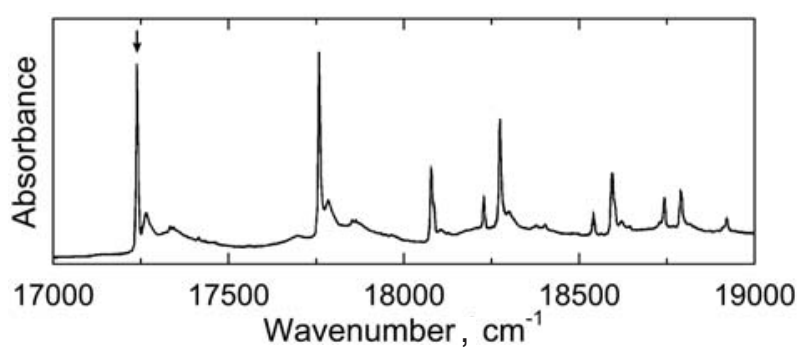

Fig. 6. Absorption spectrum of DMST- $h_{6}$ in $n$-heptane- $h_{16}$.

the influence of an external electric field. This splitting is a characteristic signature that the molecule has acquired a dipole moment in the host lattice. Accordingly, the two methyl groups are no longer equivalent. Figure 8 shows how the hole spectrum develops with time: as time progresses, sideholes grow in and the central hole fills up. Obviously the holeburning mechanism in this system is not related to the tunneling states, in contrast to the $n$-hexane case. However, once the hole is there, we can observe tunneling relaxation through the growth of sideholes, as discussed above. Figure 9 shows the tunneling relaxation rate for DMST- $h_{6}$ in $n$-hexane- $h_{16}$. There are several noteworthy features: between 4 and $14 \mathrm{~K}$ the tunneling relaxation rate follows a Raman process. A perfect $T^{7}$ dependence should result in a straight line in a log-log representation. However, there is a slight bend over to a somewhat weaker temperature dependence. This is due to the very low Debye temperature in this case, which is about $32 \mathrm{~K}$. Below about $3 \mathrm{~K}$ the thermal behavior of the rate levels off to a direct process. Since the Stark spectra (Fig. 7) tell us that the methyl groups are not equivalent, we

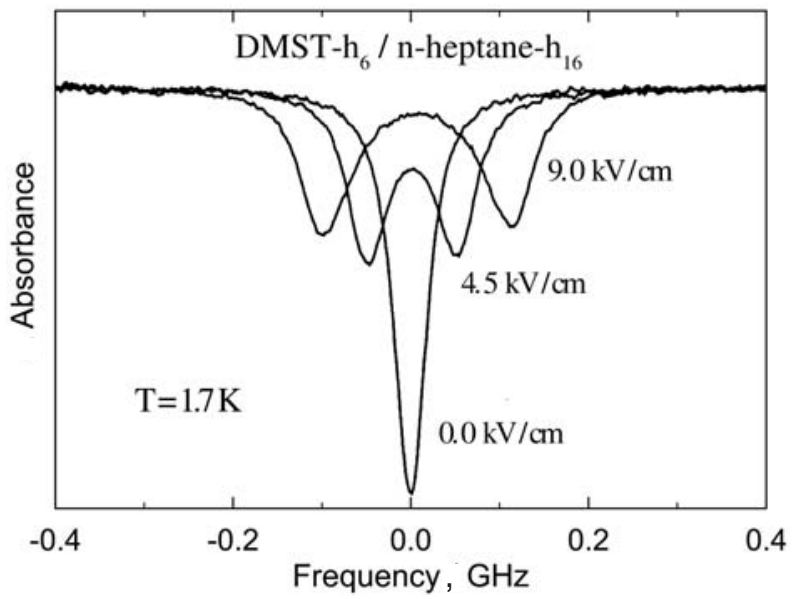

Fig. 7. Stark effect on the central hole. The splitting is a signature for a broken inversion symmetry of DMST and hence, for non-equivalent methyl groups. 


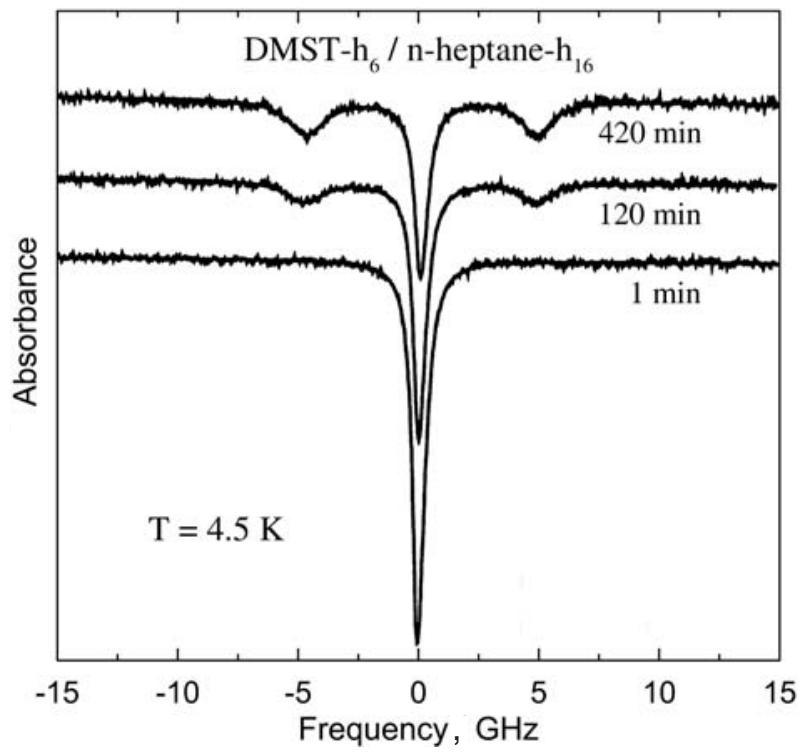

Fig. 8. Evolution of the hole-burning spectrum of DMST- $h_{6}$ in $n$-heptane- $h_{16}$ as a function of waiting time. Note the growing-in of sideholes.

expected to observe 4 sideholes instead of two. However, what we could not observe with DMST- $h_{6}$ for reasons we do not know, was indeed observed for DMST- $d_{6}$ in the $n$-heptane- $d_{16}$ matrix.

Figure 10 shows the evolution of the hole-burned spectrum with waiting time. This pattern is very peculiar, indeed: immediately after burning we observe two sideholes and two antiholes apart from the central hole. However, as time evolves, the antiholes vanish and turn into sideholes, while the central hole progressively fills. In addition, the originally present sideholes grow, but after some time, they stop growing and, instead, start decaying. Eventually, at very long waiting times, a third pair of sideholes grows in.

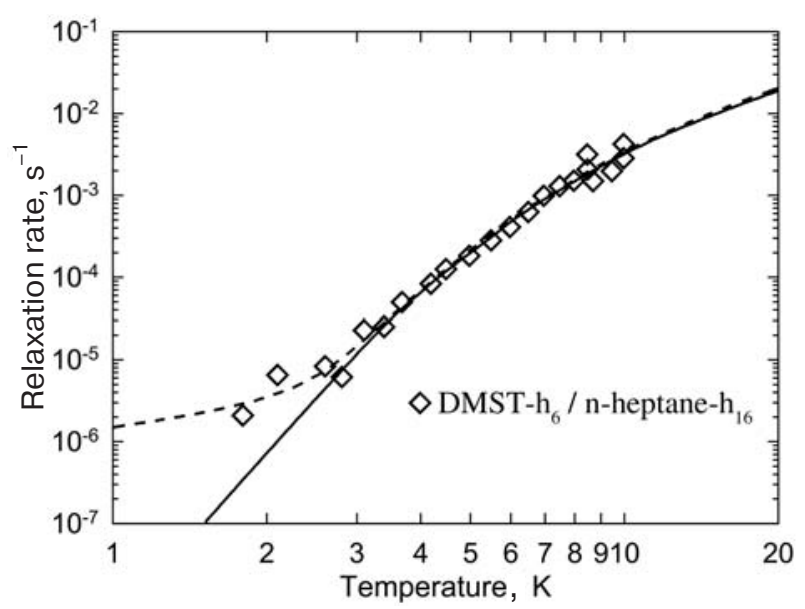

Fig. 9. Tunneling relaxation rate of $\mathrm{DMST}^{-h_{6}}$ in $n$-heptane- $h_{16}$ as a function of temperature.

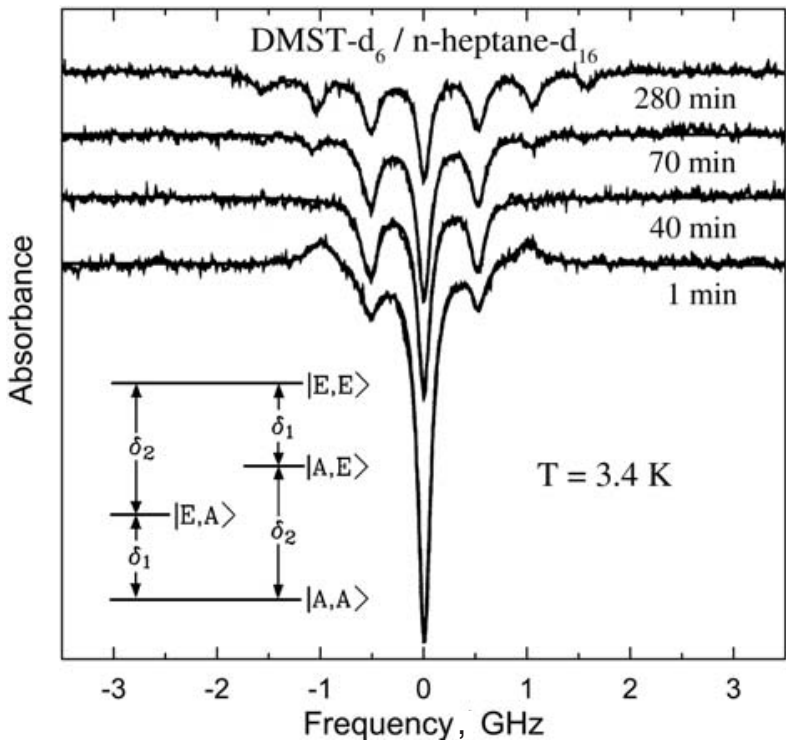

Fig. 10. Time evolution of the hole-burned spectrum of DMST- $d_{6}$ in $n$-heptane- $d_{16}$ at $3.4 \mathrm{~K}$. Inset: level scheme for 2 non-equivalent methyl groups.

The inset in Fig. 10 shows the level scheme, which is based on the assumption of two different and independent methyl groups. In this scheme there are 4 possible states, namely the $A A$ state in which both groups are in their ground state, the $E A$ and the $A E$ states in which either the left or the right methyl group is excited, and finally, the $E E$ state in which both groups are excited. A similar level structuring occurs in the electronically excited state. This level scheme gives rise to three transitions with characteristic frequency spacings $\left|\delta_{1}-\delta_{1}^{*}\right|,\left|\delta_{2}-\delta_{2}^{*}\right|$, and $\left|\left(\delta_{1}+\delta_{2}\right)-\left(\delta_{1}^{*}+\delta_{2}^{*}\right)\right|$. These level spacings imply that the distance from the central hole to the first sidehole is exactly equal to the distance from the second sidehole or antihole to the third, in perfect agreement with the experimental observation.

The dynamics of the various holes at a temperature of $3.4 \mathrm{~K}$ is shown in Fig. 11. From the above level scheme we expect three rate constants, namely $2 k_{1}$, $2 k_{2}$ and $2\left(k_{1}+k_{2}\right)$. However, the experiment tells us that one methyl group is much faster than the other, say $k_{2}<<k_{1}$, then the three rate constants reduce to two in agreement with what is measured. Consider, for instance, the first side hole. It grows quickly with time at short waiting times. We associate it with the relaxation of the $E A$ state. The relaxation rate of this state is assigned to be $k_{1}$. As the waiting time progresses, the $E A$ state becomes progressively repopulated due to relaxation from the $E E$ state with the slow rate constant $k_{2}$. Hence, hole growth stops, and then starts refilling. The peculiar observation that an antihole turns into a sidehole is due to the fact that the 


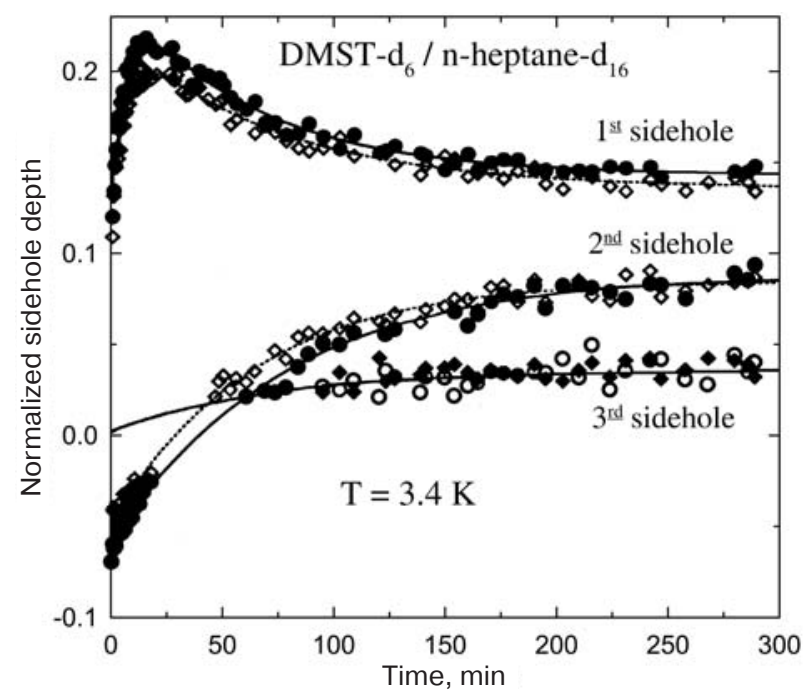

Fig. 11. Time evolution of the hole depth for the various sideholes shown in Fig. 10. The fit curves are monoexponentials except for the first sidehole, which grows in with rate constant $k_{1}$ before subsequently decreasing with rate constant $k_{2}$. Open and filled symbols refer to the high and low frequency side features.

phototransformation is not unique but is mixed partly due to a conformational change in the host lattice around the probe, and partly due to an optically induced symmetry conversion within the tunneling levels in the excited triplet state.

\section{DMST in n-octane}

We did quite a few experiments with DMST in $n$-octane. As a matter of fact, this was the system where we performed the first dynamic experiments.

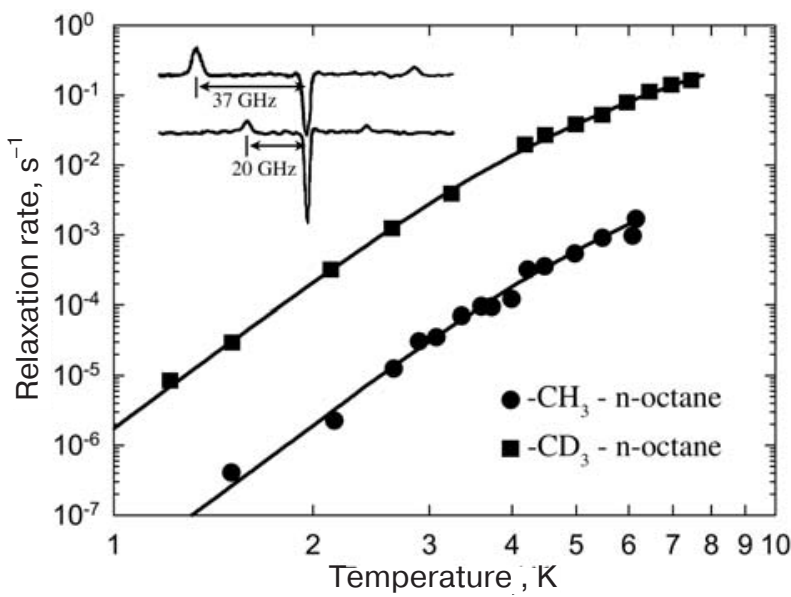

Fig. 12. Tunneling relaxation rate as measured through the recovery of the central hole as a function of temperature for DMST- $h_{6}$ and DMST- $d_{6}$ in $n$-octane- $h_{18}$. Deuteration speeds up relaxation by 2 orders of magnitude. The inset shows the reduction of the sidehole splitting through deuteration.
Here we are going to focus on two aspects only, namely on the very unusual effect of deuteration on the dynamics, and on the effect of breaking the permutation symmetry by asymmetric substitution of one hydrogen by a deuterium.

Figure 12 shows the tunneling relaxation rate as measured through the recovery of the central holes of DMST- $h_{6}$ and DMST- $d_{6}$ in $n$-octane- $h_{18}$. Both systems follow exactly the same temperature pattern which perfectly fits a Raman process below about $6 \mathrm{~K}$. However, there is a totally unexpected feature: throughout the Raman regime, the relaxation rate for the deuterated rotor is two orders of magnitude larger than the rate of the protonated rotor. The inset in Fig. 12 shows the hole-antihole splitting for the two samples. It is quite interesting to note that this splitting is reduced in the deuterated rotor by almost $\frac{1}{2}$, which is the expected effect in the case when the rotor is free.

How can we explain the peculiar isotope effect in the deuterated rotor? The reason is twofold: on the one hand it is due to the fact that the relaxation is governed by a Raman process. Otherwise we would not observe anything so drastic. On the other hand, it is also due to the different symmetry breaking interaction in the deuterated rotor: the nuclear quadrupole interaction. This interaction introduces an additional matrix element into the expression for the relaxation rate with the special property that it is almost independent of the height of the 3 -fold potential barrier. Accordingly, the relaxation rate remains mostly unchanged, even in the free-rotor case, whereas the matrix elements originating from the nuclear spin-spin interaction vanish in the free-rotor case. The consequence is that the ratio of the rates $k_{\mathrm{D}} / k_{\mathrm{H}}$ for a Raman process diverges as the rotor becomes free. In the case of DMST in $n$-octane this ratio is about 100. This number nicely fits the observed reduction of the sidehole splitting by almost a factor of 2 (Fig. 12, inset) from which we conclude that the rotor in $n$-octane is almost free [22].

We now consider the change in tunneling dynamics if the symmetry of the rotor is broken by asymmetric deuteration. Already from the broad band spectra (Fig. 13) we see that something specific does occur: the band becomes structured and, in addition, becomes strongly temperature dependent. Hole-burning results in a complicated pattern of at least 6 sideholes whose depths, however, no longer depend on waiting time. Lowering the temperature to $1.5 \mathrm{~K}$ reduces the intensity of the high frequency peak strongly and also reduces the number of sideholes to 2 . Since there are no symmetry restrictions anymore, the degeneracy of the $E$-state is lifted, resulting in 3 levels with spacings 


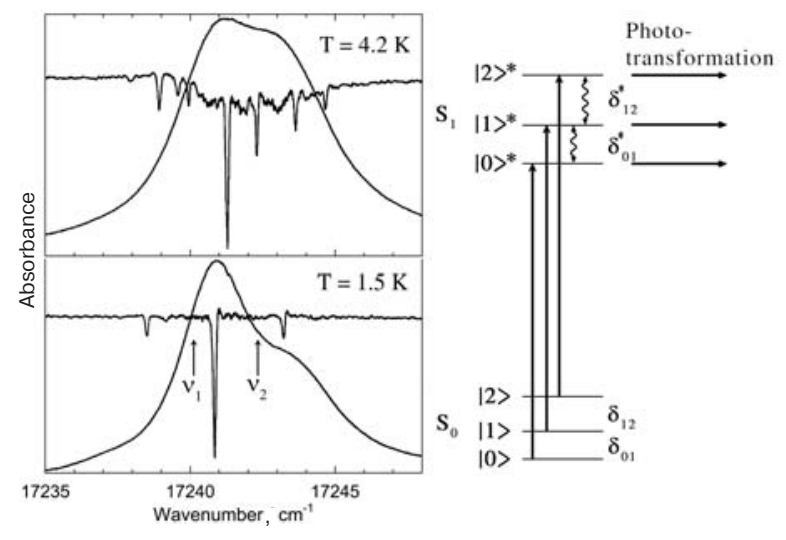

Fig. 13. The region of the electronic 00-absorption together with the hole-burned spectra for DMST- $h_{4} d_{2}$ in $n$-octane- $h_{18}$ at 4.2 and $1.5 \mathrm{~K}$. The diagram on the right side shows rotor levels and the associated optical transitions.

$\delta_{01}, \delta_{02}$ and $\delta_{01}+\delta_{02}$. A similar level pattern occurs in the excited state. Hole-burning must be solely due to a conformational change in the lattice because population can no longer be stored in the tunneling states. These tunneling states are, in contrast to the symmetric rotor, connected by fast relaxation processes. Because of the unfavorable Franck-Condon factors, transitions between different tunneling states are highly forbidden. Therefore, as sketched on the rightside of Fig. 13, we have 3 transitions with frequency splittings $\left|\delta_{01}-\delta_{01}^{*}\right|,\left|\delta_{12}-\delta_{12}^{*}\right|$, and $\left|\left(\delta_{01}+\delta_{12}\right)-\left(\delta_{01}^{*}+\delta_{12}^{*}\right)\right|$. Suppose we excite the $1^{*} \leftarrow 1$ transition and bleach a certain frequency position in its associated inhomogeneous band. Then, since the levels are in fast equilibrium, population from the same sites in the inhomogenous bands of the

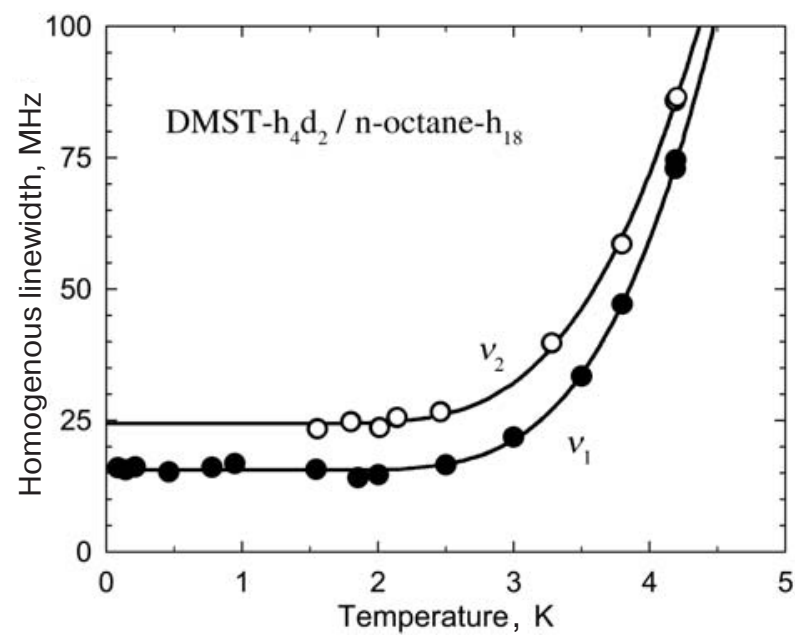

Fig. 14. The homogenous linewidth of the 00-transition as a function of temperature as measured at the two frequencies marked $v_{1}$ and $v_{2}$ in Fig. 13. other two transitions will be transferred to balance the loss of population through bleaching. For three overlapping transitions we expect six sideholes in addition to the central hole. The observed pattern at $4.2 \mathrm{~K}$ comes close to this expectation. Lowering the temperature to $1.5 \mathrm{~K}$ may depopulate level 2 so that we have only two overlapping bands. Accordingly, there will be only two sideholes, in agreement with the experimental results.

In order to get an idea how fast the dynamics among the tunneling levels without symmetry restriction is, we measured the homogeneous linewidth at the positions marked $v_{1}$ and $v_{2}$ in the inhomogeneous spectrum (Fig. 13) [23]. From the temperature dependence, shown in Fig. 14, we know that the higher frequency $v_{2}$ corresponds to a higher energy tunneling level. As $T$ approaches zero, there is a constant difference, $\Delta \gamma_{h}(T=0)$, between the homogeneous linewidth of the two transitions of about 8.7 MHz. This difference, of course, comes from the relaxation times in the ground and excited state connecting levels 0 and $1, \tau_{01}$ and $\tau_{01}^{*}$. Assuming that both lifetimes are equal we can estimate them from the measured difference in the linewidth to be about $0.04 \mu \mathrm{s}$. Comparing this with the corresponding lifetime at around $1.5 \mathrm{~K}$ of the symmetric protonated rotor, we find that the breakdown of the permutation symmetry speeds up relaxation by about 14 orders of magnitude.

\section{PCT in cyclohexane}

One problem with the optical experiments on rotational tunneling relaxation is that they do not enable direct access to the most important parameter in the respective dynamics, namely the tunneling splitting $\delta$. A simple variation of the temperature does not change the situation either, because the levels are not in thermal equilibrium. In the following we show how, despite these shortcomings, the tunneling splitting in the ground as well as in the excited state, can be measured. Knowing these parameters, everything (rotational barriers and rotational eigenvalues in the ground and the excited states) can be determined from a solution of the Mathieu equation [24]. The experiments were performed with PCT in cyclohexane.

Figure 15 shows the absorption spectrum of PCT in cyclohexane at $4 \mathrm{~K}$. The arrow marks the transition where the hole-burning experiments have been performed. A highly resolved spectrum around the marked frequency together with an overlaid holeburned spectrum is shown on the right hand side of the figure. The temperature dependence of the tunneling relaxation rate, as obtained from measuring the recovery of the central hole, is shown in Fig. 16. For a 


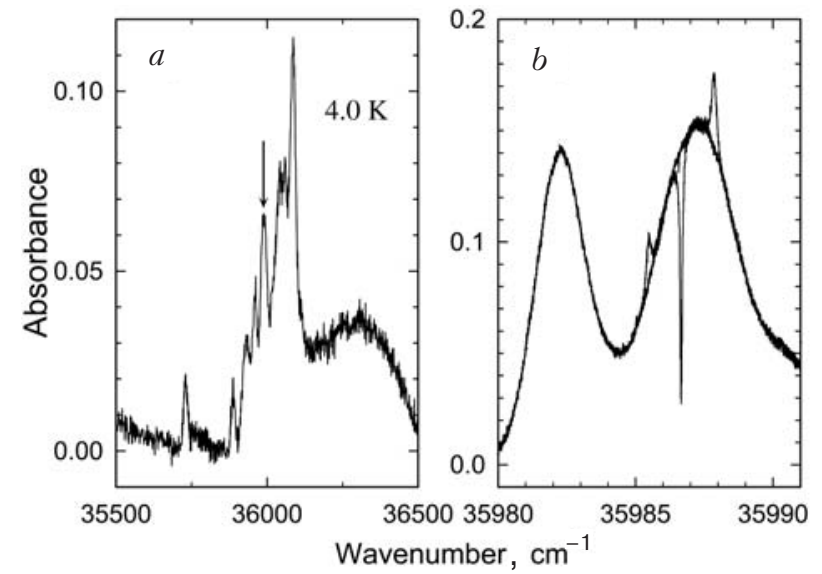

Fig. 15. Absorption spectrum of $p$-chlorotoluene (PCT) in cyclohexane at $4 \mathrm{~K}$. The arrow marks the band where hole-burning was performed. The right hand side shows the indicated region of the spectrum at high resolution with an overlaid hole-burned spectrum.

safe interpretation of the associated processes, the tunneling splitting has to be known. We measured it in the following way: first of all, the relaxation time as a function of temperature has to be known. As shown in the many examples above, it can be measured with high accuracy in a straightforward way. In the present case we measured it between 3 and $16 \mathrm{~K}$ (Fig. 16). The next step concerns the integrated intensity of the two antiholes. They reflect the population difference of the $A$ and $E$ levels in the ground state. At sufficiently high temperatures, where the relaxation is fast enough compared to the experimental time scale, this difference is given by the Boltzmann factor $p\left(T_{e}\right)=\exp \left(-\delta / k_{B} T_{e}\right)$ at the respective temperature $T_{e}$. So we raise the temperature to $T_{e}$ and wait sufficiently long for equilibration. The

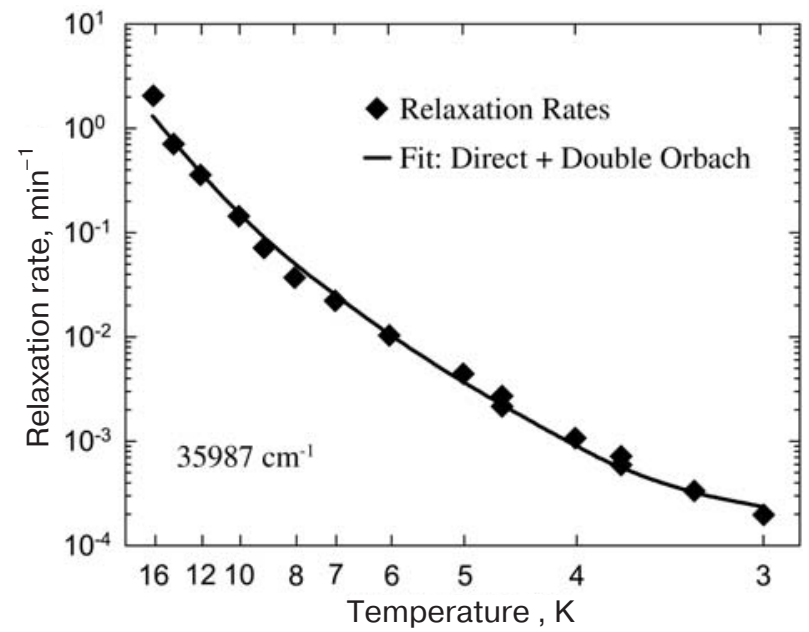

Fig. 16. Tunneling relaxation rate as a function of temperature. The fit curve is based on a double Orbach process plus a direct process. See text for details. proper waiting time can be deduced from the relaxation times. We chose $9 \mathrm{~K}$ for $T_{e}$. The relaxation time at $T_{e}$ is about $15 \mathrm{~min}$. As a proper equilibration time we chose at least one hour. Then, we cooled the system quickly to a temperature $T_{r}$, in our case $5 \mathrm{~K}$. The respective relaxation time is $\tau_{r}$. There, we let it relax for a variable waiting time $t$. To measure the intensity ratio of the antiholes after a waiting time $t$, the temperature was further lowered to $3 \mathrm{~K}$ to ensure that the population did not change during the measuring time. Our goal is to determine the population ratio $N_{A}(t) / N_{E}(t)$ at $T_{r}$. This population is governed by the simple equation:

$$
\frac{d N_{A}(t)}{d t}=-\frac{1}{\tau_{r}}\left[N_{A}-N_{A}(\infty)\right] .
$$

and similarly for $N_{E}$. The solution of these equations yield the population ratio:

$$
\begin{gathered}
\frac{N_{A}(t)}{N_{E}(t)}= \\
=P \frac{1+p\left(T_{e}\right)+\left[p\left(T_{r}\right)-p\left(T_{e}\right)\right] \exp \left(-t / \tau_{r}\right)}{p\left(T_{r}\right)\left[1+p\left(T_{e}\right)\right]-\left[p\left(T_{r}\right)-p\left(T_{e}\right)\right] \exp \left(-t / \tau_{r}\right)} .
\end{gathered}
$$

$P$ is a scaling parameter which does not depend on $\tau_{r}$ or $t$. The above equation is, via the Boltzmann factors, an equation for $\delta$. Figure 17 shows a fit of this equation to the intensity ratio of the antiholes as a function of waiting time. From the fit, $\delta$ was determined to be $(117 \pm 4) \mathrm{GHz}$. Using the observed hole-antihole splitting of $33 \mathrm{GHz}, \delta^{*}$ was calculated as $84 \mathrm{GHz}$. Using these results, $V_{3}$ and $V_{3}^{*}$ as well as the associated librational frequencies could be determined by solving the Mathieu equation. The result is $(833 \pm 60) \mathrm{GHz}$ and $(1370 \pm 200) \mathrm{GHz}$ for the barrier heights, respectively. The lowest librational energies

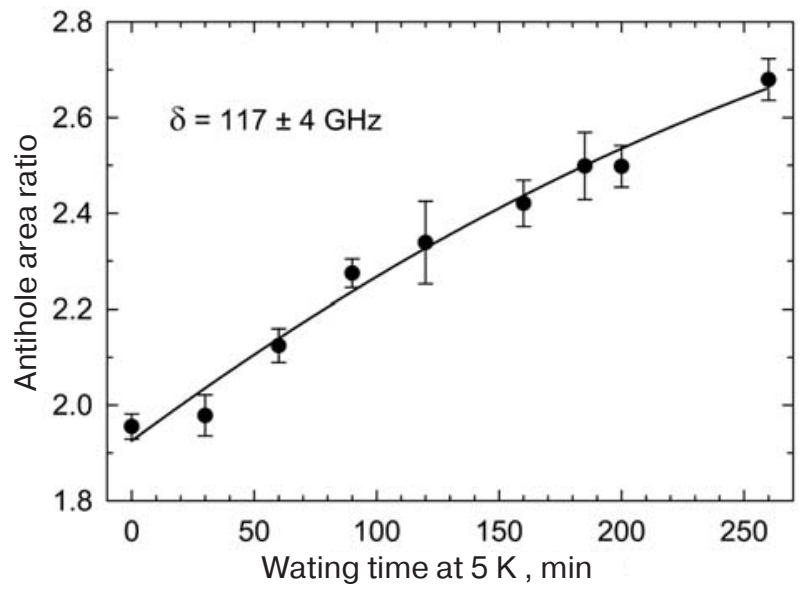

Fig. 17. The intensity ratio of the two antiholes as a function of waiting time at $5 \mathrm{~K}$. The fit curve is based on equation (3). 
in the electronic ground state $E_{1}^{E}-E_{0}^{E}$ and $E_{1}^{A}-E_{0}^{A}$ were calculated to be $4.13 B$ and $9.28 B$, respectively, where $B$ is the rotational constant of the free methyl rotor. On the basis of these numbers a precise interpretation of the relaxation data (Fig. 16) is possible: the fit curve is based on a double Orbach-process with the above activation energies, plus a direct process which comes in below about $4 \mathrm{~K}$. The prefactors of the two Orbach processes are 79 and $1.7 \mathrm{~min}^{-1}$, respectively.

\section{Summary}

Hole-burning techniques enable deep insight into the rotational tunneling dynamics of methyl groups with unprecedented accuracy and provide detailed information on a large body of microscopic parameters. This high accuracy is due to the fact that hole-burning has the potential to induce nuclear spin polarization (in the $\mathrm{CH}_{3}$-rotor) or symmetry species conversion (in the $\mathrm{CD}_{3}$-rotor) on a macroscopic level just by photobleaching a certain spectral site by nearly $100 \%$. The key which gives rise to the quantum effects is the inherent symmetry of even permutations which puts tremendous restrictions on the relaxing tunneling levels via Pauli's principle. We could show that a breakdown of this symmetry speeds up the dynamics by about 14 orders of magnitude. Many of the measured features, for instance the peculiar isotope effect observed for DMST in $n$-octane, could be interpreted on a quantitative basis. All processes predicted by theory could be observed. However, there are also some mysteries for which we could not find any reasonable explanation. Methyl groups attached to proper chromophores are extremely sensitive probes for the solid state environment in which they are embedded. Hence, measuring rotational tunneling relaxation is not only an interesting field in molecular physics, but also in solid state physics.

We acknowledge support from the DFG (SFB 533, B5) and from the Fonds der chemischen Industrie.
1. W. Press, in: Springer Tracts in Modern Physics, Vol. 92, G. Höhler (ed.), Springer, Berlin (1981).

2. A. Hüller, Phys. i.u. Zeit. 3, 131 (1996).

3. W. Press, A. Heidemann, H. Lauter, et al., Can. J. Chem. 66, 686 (1988).

4. A. Heidemann, K.J. Lushington, J.A. Morrison, et al., J. Chem. Phys. 81, 5799 (1984).

5. K. Guckelsberger, H. Friedrich, and R. Scherm, Z. Phys. B91, 209 (1993).

6. G. Vandemaele, P. Coppens, and L. Vangerven, Phys. Rev. Lett. 56, 1202 (1986).

7. A. Buekenhoudt, G. Vandemaele, and L. Van Gerven, Phys. Rev. B41, 9038 (1990).

8. A. Inaba, H. Chihara, J. A. Morrison, et al., J. Phys. Soc. Jpn. 59, 522 (1990).

9. M. Plazanet, M.A. Neumann, and H.P. Trommsdorff, Chem. Phys. Lett. 320, 651 (2000).

10. C. Borczyskowski, A. Oppenländer, H.P. Trommsdorff, et al., Phys. Rev. Lett. 65, 3277 (1990).

11. G. Gradl, K. Orth, and J. Friedrich, Europhys, Lett. 19, 459 (1992)

12. G. Gradl, A. Feis, and J. Friedrich, J. Phys. Chem. 96, 2080 (1992).

13. W. Häusler, Z. Phys. B81, 266 (1990).

14. A. Würger, Z. Phys. B81, 273 (1990).

15. G. Diezemann and W. Häusler, J. Phys. Cond. Matt. 5, 6121 (1993).

16. J. Friedrich and D. Haarer, Ang. Chem. Int. Ed. 23, 113 (1984).

17. H. Maier, B.M. Kharlamov, and D. Haarer, in: Tunneling Systems in Amorphous and Crystalline Solids, P. Esquinazi (ed.), Springer, Berlin (1998).

18. K.K. Rebane and L.A. Rebane, in: Persistent Spectral Hole-Burning: Science and Applications, Vol. 44, W.E. Moerner (ed.), Springer, Berlin (1988).

19. R. M. Dimeo, Am. J. Phys. 71, 885 (2003).

20. K. Orth, F. Rohlfing, and J. Friedrich, Z. Phys. B95, 493 (1994)

21. V. Gebhardt, K. Orth, and J. Friedrich, J. Chem. Phys. 104, 942 (1996).

22. K. Orth, P. Schellenberg, J. Friedrich, et al., J. Lumin. 56, 99 (1993).

23. M. Johnson, K. Orth, J. Friedrich, et al., J. Chem. Phys. 105, 9762 (1996).

24. M. Pinsker and J. Friedrich, J. Chem. Phys. 117, 4639 (2002). 